\title{
Multi-Agent Motion Planning Using Bayes Risk
}

\author{
Matthew J. Bays ${ }^{\mathrm{a}, 1,3, *}$, Apoorva Shende ${ }^{\mathrm{a}, 2}$, Daniel J. Stilwell ${ }^{\mathrm{a}, 2,4, * *}$ \\ ${ }^{a}$ Blacksburg, VA 24060
}

\section{Abstract}

We introduce a novel approach to controlling the motion of a team of agents so that they jointly minimize a cost function utilizing Bayes risk. Bayes risk is a useful measure of performance for applications where agents must perform a classification task, but is often difficult to compute analytically for many applications involving agent state variables. We use a particlebased approach that allows us to approximate Bayes risk and express the optimization problem as a mixed-integer linear program. By minimizing Bayes risk, agents are able to account explicitly for the costs associated with correct and incorrect classification. We illustrate our approach with a target interception problem in which a team of mobile agents must intercept mobile targets that are likely to enter a specified area in the near future. We show that the cooperative agent motion that minimizes a cost function utilizing Bayes risk is an efficient way to achieve selective interception.

\footnotetext{
*Corresponding author

** Principal corresponding author Email addresses: mjb222@ieee.org (Matthew J. Bays), apoorva@vt.edu (Apoorva Shende), stilwell@vt.edu (Daniel J. Stilwell)

${ }^{1}$ Naval Surface Warfare Center, Panama City Division

${ }^{2}$ Bradley Department of Electrical \& Computer Engineering, Virginia Tech

${ }^{3}$ Tel/Fax: 561-818-1765

${ }^{4}$ Tel/Fax: 540-231-3204/540-231-3362
} 
Keywords:

Autonomous Agents, Robotics, Motion Planning, Bayes Risk, Classification, Decision Theory

\section{INTRODUCTION}

We investigate a method for coordinating the motion of a group of agents or mobile sensors to improve performance of a classification task. We propose a particle-based approximation of Bayes risk in order to develop a cost function to guide the motion of the agents. Bayes risk is the prior-averaged cost incurred by a decision rule [1]. Bayesian probabilistic techniques are particularly relevant for classification-based feedback control since they inherently update the probabilistic distribution of the underlying state as new observations become available. The updated state distribution that is computed using Bayesian filtering techniques can be used to compute the Bayes risk corresponding to the current observations. Prior work on cooperative control has often addressed optimal sensor motion for improved sensing performance, as in the case of maximizing information [2]. In typical cases, sensor motions produce optimal state estimates, but would not necessarily lead to optimal, or even improved, classification performance. The research into the decision-making and classification has largely been the work of the statistics and signal processing community, where an optimal decision rule is generated for the observations already gathered. A good example of these contributions in the area of target classification is [3]. The primary contri-

This paper was presented in part at the IEEE International Conference on Robotics and Automation, St. Paul, MN, May 2012. 
bution of our work is attempting to bridge the gap between classification and agent motion control (e.g. cooperative control as in [4]) by proposing a methodology for which cooperative sensor motion is used to intentionally improve classification performance. We provide context for our Bayes riskbased motion control using a target interception application.

Bayes risk has been used frequently in the signal processing community; see for example $[5,6,7,8]$. However, its use in motion planning has been limited. Blackmore et al in [9] develop an approach for model selection from a family of dynamic systems using a special case of Bayes risk that is equivalent to the probability of error. In their approach, control signals for the dynamic system are computed that minimize an upper bound for the probability of error. We generate control signals that affect the paths for a team of mobile agents. However, we choose the control signals to minimize an approximation to a more general form of Bayes risk.

The problem of area protection has been studied using a variety of techniques. Smith uses formal geometric approaches in [10] to choose paths for vehicles servicing randomly generated targets whose locations are deterministically known. In contrast, our particle-based solution to target interception takes into account uncertainty in target locations at every time-step. Agmon develops a novel patrol scheme in order to minimize the probability of adversary penetration in [11]. Our work complements Agmon's by addressing the same scenario, but optimizing the agents' reactions to incoming targets instead of optimizing the standard patrol pattern. Beard uses a $k$-best paths graph search to intercept deterministic targets within an area in [12]. Like our work, Earl and D'Andrea studied the target interception problem in 
a MILP framework [13]. However, we introduce a formal risk metric, and generalize system dynamics from a deterministic framework to incorporate stochastic dynamics and noise. Additionally, we formulate the problem using a receding-horizon control approach, limiting the planning horizon to a subset of the total mission time and thus complexity of the problem. Chasparis and Shamma solve the problem of area protection in [14] using a discrete-time, discrete-space resource allocation framework. Control policies are modeled as the transition of resources from cell to cell. The objective of maximizing the interceptions of enemy vehicles is modeled using a linear cost function. In contrast, our work explicitly addresses Bayesian risk. Shende solves the problem of stochastic target interception using closed-form properties of the Gaussian distribution in [15] for instances where the system noise is normally distributed, however does not use Bayes risk as a cost function.

The use of Bayes risk for collision avoidance has been previously demonstrated by Jansson and Gustafsson for automobile applications in [16]. In their work, a binary decision rule was implemented. Their decision rule was optimal in the Bayes risk sense, and if met, a collision avoidance maneuver was executed. Our work explicitly couples agent motion control to a cost function utilizing Bayes risk, and as a result, minimizes the risk of a collision.

This paper is outlined as follows. We present the general problem formulation we use for our agent-based motion control and target interception, its relation to Bayes risk, and our framework's underlying assumptions in Section 2. We then propose a particle-based approximation for calculating the necessary probabilities and thereby Bayes risk in Section 3. We extend the particle framework to incorporate collision avoidance in Section 4. Specific 
estimation techniques used in order to create the particle framework, and a discussion of the potential impact of assumptions on real implementations are provided in Section 5. Finally, we present simulation results of the framework using the interception-only model as well as the collision avoidance extension in Section 6.

\section{PROBLEM FORMULATION \& BAYES RISK}

In our application of target interception, $M$ cooperative agents and $N$ targets maneuver within a convex area $G \subset \mathbb{R}^{2}$. The agents must prevent the targets from entering a subset $A \subset G$ by selectively intercepting those targets that will enter the area of protection. There is uncertainty associated with initial target and agent positions, as well as their dynamics. Additional uncertainty is also associated with target measurements from sensors on-board the agents. The problem is to develop a control law over the optimization interval $[t, t+T]$ to guide the cooperative agents such that they intercept all the targets that would have entered the area of protection if not intercepted, and to avoid taking action against targets that would not have entered the area. Additionally, we assume that the targets are not adversarial. That is, the targets do not perform control actions taking into account the states of the agents.

\subsection{The Target Interception Problem}

We will now define the decision problem with respect to motion planning and classification. At each time $\tau \in[t, t+T]$ within the optimization interval, we consider a target trajectory over the interval $\left[\tau, \tau+T^{\prime}\right]$. We will call this horizon the threat assessment interval. Note that the threat assessment 
horizon $T^{\prime}$ and planning horizon $T$ are independently specified. The trajectory of target $i$ is classified to be within the set of trajectories considered a threat, or the set of trajectories considered not a threat. The event that the trajectory of target $i$ over the interval $\left[\tau, \tau+T^{\prime}\right]$ is a threat is denoted as $\Omega_{i}(\tau)$. The event that the target trajectory is within the set of trajectories considered not a threat is denoted as $\sim \Omega_{i}(\tau)$. Target $i$ is classified as a threat if it enters the area of protection $A$ during this interval. A correct decision that should be reflected through the agent control actions is to intercept the target. This decision is denoted $\Gamma_{i}(\tau)$ for target $i$ at time $\tau$. A trajectory that does not enter an area of protection within the time horizon $\left[\tau, \tau+T^{\prime}\right]$, or has been intercepted by an agent before $\tau$, is categorized as not a threat $\left(\sim \Omega_{i}(\tau)\right)$, and corresponds to a correct decision of non-interception $\left(\sim \Gamma_{i}(\tau)\right)$. For a target, the decision rule that must hold true for the target interception problem may be written

$$
\begin{gathered}
\Omega_{i}(\tau) \rightarrow \Gamma_{i}\left(\tau^{\prime}\right) \\
\sim \Omega_{i}(\tau) \rightarrow \sim \Gamma_{i}\left(\tau^{\prime}\right)
\end{gathered}
$$

for some time $\tau^{\prime}<\tau \in[t, t+T]$. However, (1)-(2) is a decision rule that would only work if the problem were purely deterministic. In order to take into account the probabilistic nature of the target interception problem, we turn to Bayes risk.

\subsection{Bayes Risk for Target Interception}

In the following sections we describe an agent control law designed to minimize the cumulative Bayes risk associated with the target interception 
problem involving the threat and interception events defined in (1)-(2). Let $R_{\Omega_{i}}(\tau)$ be the cost incurred conditioned on hypothesis $\Omega_{i}$ being true at time $\tau$. In Bayes risk terminology, $R_{\Omega_{i}}(\tau)$ is often called the conditional risk associated with the hypothesis $\Omega_{i}(\tau)$ being true. Similarly, $R_{\sim \Omega_{i}}(\tau)$ is the conditional risk associated with the hypothesis $\sim \Omega_{i}(\tau)$ being true at time $\tau$. The Bayes risk at time $\tau$ is then given by

$$
R_{i}(\tau)=R_{\Omega_{i}}(\tau) P\left(\Omega_{i}(\tau)\right)+R_{\sim \Omega_{i}}(\tau) P\left(\sim \Omega_{i}(\tau)\right)
$$

where $P\left(\Omega_{i}(\tau)\right)$ and $P\left(\sim \Omega_{i}(\tau)\right)$ are the priors of the two classes at time $\tau$. The conditional risks $R_{\Omega_{i}}(\tau)$ and $R_{\sim \Omega_{i}}(\tau)$ are written

$$
\begin{gathered}
R_{\sim \Omega_{i}}(\tau)=C_{0,0} P\left(\sim \Gamma_{i}(\tau) \mid \sim \Omega_{i}(\tau)\right)+C_{1,0} P\left(\Gamma_{i}(\tau) \mid \sim \Omega_{i}(\tau)\right) \\
R_{\Omega_{i}}(\tau)=C_{0,1} P\left(\sim \Gamma_{i}(\tau) \mid \Omega_{i}(\tau)\right)+C_{1,1} P\left(\Gamma_{i}(\tau) \mid \Omega_{i}(\tau)\right),
\end{gathered}
$$

where $C_{0,0}, C_{1,0}, C_{0,1}$ and $C_{1,1}$ are user-selected coefficients associated with the cost of correct and incorrect classification. From the standard definition of Bayes risk, $C_{0,0}$ and $C_{1,1}$ are the costs of correct classification, and $C_{1,0}$ and $C_{0,1}$ are the costs of false-alarm and missed detection, respectively [1]. If we assume $\Omega_{i}(\tau)$ and $\Gamma_{i}(\tau)$ to be independent events for a given control action, and then from (3) and (4) Bayes risk can be expressed

$$
\begin{aligned}
R_{i}(\tau)= & C_{0,1} P\left(\sim \Gamma_{i}(\tau)\right) P\left(\Omega_{i}(\tau)\right)+C_{1,1} P\left(\Gamma_{i}(\tau)\right) P\left(\Omega_{i}(\tau)\right) \\
& \left.+C_{0,0} P\left(\sim \Gamma_{i}(\tau)\right) P\left(\sim \Omega_{i} \tau\right)\right)+C_{1,0} P\left(\Gamma_{i}(\tau)\right) P\left(\sim \Omega_{i}(\tau)\right) .
\end{aligned}
$$


The costs $C_{0,0}$ and $C_{1,1}$ are costs for correct classification, and are often set to zero. The misclassification costs $C_{1,0}$ and $C_{0,1}$ on the other hand are typically set to relatively high positive values and will have a significant influence on the decision rule. A relatively high false-alarm cost $\left(C_{1,0}\right)$ will cause the system to only react with control actions resulting in $\Gamma(\tau)$. A high missed-detection cost $\left(C_{0,1}\right)$ will cause the system to intercept targets that have a relatively small chance of being a threat. Thus, the use of Bayes risk as a cost function allows for the missed-detection to false-alarm cost ratio to be specified by the user. When the costs of correct classification $C_{0,0}=C_{1,1}=0$, the costs of incorrect classification $C_{1,0}=C_{0,1}=1$, and the Bayes risk is properly normalized, then it becomes the average probability of error, as defined in [17]. Throughout this work we use the general case of arbitrary costs. The optimal vehicle control actions, and resulting decision between $\Gamma_{i}(\tau)$ and $\sim \Gamma_{i}(\tau)$ at time $\tau$ are then those that collectively minimize Bayes risk.

The key to minimizing (5) is to have a means to evaluate the probabilities $P\left(\Omega_{i}(\tau)\right), P\left(\sim \Omega_{i}(\tau)\right), P\left(\Gamma_{i}(\tau)\right)$, and $P\left(\sim \Gamma_{i}(\tau)\right)$. Using a traditional Bayes decision framework, the probabilities are typically written

$$
\begin{aligned}
P\left(\Omega_{i}(\tau)\right) & =\int_{\mathbf{x} \in \Omega_{i}^{s}} p(\mathbf{x} \mid \hat{\mathbf{x}}(\tau)) d \mathbf{x} \\
P\left(\sim \Omega_{i}(\tau)\right) & =1-P\left(\Omega_{i}(\tau)\right) \\
P\left(\Gamma_{i}(\tau)\right) & =\int_{\mathbf{x}, \mathbf{s} \in \Gamma_{i}^{s}} p(\mathbf{x}, \mathbf{s} \mid \hat{\mathbf{x}}(\tau), \hat{\mathbf{s}}(\tau), \mathbf{u}(t)) d \mathbf{x} d \mathbf{s} \\
P\left(\sim \Gamma_{i}(\tau)\right) & =1-P\left(\Gamma_{i}(\tau)\right)
\end{aligned}
$$


where $\hat{\mathbf{x}}(\tau)$ and $\hat{\mathbf{s}}(\tau)$ are current measurements of the target and agent states within the planning interval, $\Omega_{i}^{s}$ is the set of target $i$ trajectories that enter the region of protection, and $\Gamma_{i}^{s}$ is the set of target $i$ and agent trajectories corresponding to interception. Evaluating the probabilities found in (6)-(9) analytically would be difficult for any system of non-trivial complication, even with the independence assumption from (4). An example is when the distributions $p(\mathbf{x} \mid \hat{\mathbf{x}}(\tau))$ and $p(\mathbf{x}, \mathbf{s} \mid \hat{\mathbf{x}}(\tau), \hat{\mathbf{s}}(t), \mathbf{u}(t))$ are assumed Gaussian, a frequent assumption in agent motion control for agent position. In this case Liouville's Theorem indicates closed-form solutions to the integral of the probability density functions (PDFs) cannot be found, and thus computational techniques must be used [18].

\subsection{Assumptions on Agent and Target Dynamics}

We now discuss the underlying assumptions on the agent and target dynamics necessary for the derivation of our Bayes risk-based motion planning framework. We assume that the motion and position of the incoming targets are governed by the form found in Assumption 2.1.

Assumption 2.1. Let $\boldsymbol{\nu}_{i}(t)$ be the process noise of target $i$ at global time $t$. Then we assume the dynamics of the target $i \in N$ follows the form

$$
\mathbf{x}_{i}(t+1)=\mathbf{f}\left(\mathbf{x}_{i}(t), \boldsymbol{\nu}_{i}(t)\right)
$$

and there exists an affine linear transformation $\mathbf{L}_{\mathbf{t a r}}(\cdot)$ such that the target position is given by

$$
\mathbf{x}_{\mathbf{p}_{i}}(t)=\mathbf{L}_{\mathbf{t a r}}\left(\mathbf{x}_{i}(t)\right)
$$


Let $\mathbf{s}_{j}(t) \in R^{m}$ be the state of agent $j \in\{1, \ldots, M\}$. We assume the motion and position of the individual agents follow the form found in Assumption 2.2 .

Assumption 2.2. Let $\boldsymbol{\mu}_{j}(t)$ be the process noise of the agent dynamics, and $\mathbf{u}_{j}(t) \in \mathbb{R}^{l_{u}}$ be the control input for agent $j$. Then we assume the agent dynamics follow the form

$$
\mathbf{s}_{j}(t+1)=\mathbf{g}_{\mathbf{j}}\left(\mathbf{s}_{j}(t), \mathbf{u}_{j}(t), \boldsymbol{\mu}_{j}(t)\right),
$$

and there exists an affine linear transformation $\mathbf{L}_{\mathbf{s e n}}(\cdot)$ such that the agent position is given by

$$
\mathbf{s}_{\mathbf{p}_{j}}(t)=\mathbf{L}_{\mathbf{s e n}}\left(\mathbf{s}_{j}(t)\right), .
$$

Additionally, our framework relies on several assumptions about the control and noise inputs for the individual agents, given in Assumption 2.3

Assumption 2.3. The elements of $\mathbf{u}_{j}$, denoted $u_{j, k}$ for $k=1, \ldots, l_{u}$, satisfy

$$
L_{j, k} \leq u_{j, k} \leq U_{j, k}
$$

for given control bounding-constants $L_{j, k}$ and $U_{j, k}$. We assume that $\mathbf{g}_{j}\left(\mathbf{s}_{j}(t), \mathbf{u}_{j}(t), \boldsymbol{\mu}_{j}(t)\right)$ in (12) is affine in $\mathbf{s}_{j}(t), \mathbf{u}_{j}(t)$, and $\boldsymbol{\mu}_{j}(t)$.

Assumption 2.3 is necessary in order to express the optimization problem as a mixed-integer linear program. No similar requirement is imposed of $\mathbf{f}_{i}(\cdot)$ in (10). Finally, we assume that estimates of the positions of the agents 
and targets are able to be communicated to a centralized planner, and the planner may communicate back the control actions to the agents.

Assumption 2.4. Target position estimate $\hat{\mathbf{x}}_{p_{i}}(t)$ and agent position estimate $\hat{\mathbf{s}}_{\mathbf{p} \mathbf{j}}(t)$ are known to a centralized motion planner, and agent control actions $\mathbf{u}_{j}(t)$ may be communicated to the individual agents at every time step $t$.

We use the above assumptions to develop our motion planning framework. In section 5.3, we discuss the practical implications of these assumptions on real implementation, as well as refinements that may mitigate their impact.

\section{PARTICLE-BASED BAYES RISK FOR AGENT MOTION CON- TROL}

We now turn to the main formulation of our particle-based Bayes risk motion planning framework. We first develop the necessary indicator variables in order to track our two primary classification and decision states: threat and interception. We then develop the particle approximations to the probabilities of the events. Finally, we formulate our cost function utilizing Bayes risk in a receding-horizon framework.

\subsection{Formulation of Indicator Variables for the Target Interception Problem}

For the target interception problem, we say that agent $j$ has intercepted target $i$ at time $\tau$ in the time horizon $[t, t+T]$ if

$$
\left.\| \mathbf{x}_{\mathbf{p}_{i}}(\tau)-\mathbf{s}_{\mathbf{p}_{j}}(\tau)\right) \| \leq R,
$$


where $R$ is a given constant. However, we note that our methodology will work for any convex polytope defining interception. We say that target $i$ is a threat at time $\tau$ if at any time in $\left[\tau, \tau+T^{\prime}\right], \mathbf{x}_{\mathbf{p}_{i}}(\tau) \in A$. The condition (15) is approximated by a regular polygon with $n_{d}$ sides. Thus the target $i$ is defined as intercepted by agent $j$ if the following set of constraints

$$
\begin{array}{r}
<\left(\mathbf{x}_{\mathbf{p}_{i}}(\tau)-\mathbf{s}_{\mathbf{p}_{j}}(\tau)\right), \mathbf{v}_{q}>\leq R \\
\text { for all } q=\left\{1, \ldots, n_{d}\right\},
\end{array}
$$

are jointly satisfied, where $\mathbf{v}_{q}=\left[\begin{array}{c}\sin \left(\theta_{q}\right) \\ \cos \left(\theta_{q}\right)\end{array}\right], \theta_{q}=\frac{2 \pi q}{n_{d}}$, and $<\cdot>$ is the inner product. We use time-dependent binary variables $b_{i j}(\tau)$ to indicate interception of target $i$ by agent $j$ at time $\tau$. We then have

$$
\begin{aligned}
b_{i j}(\tau)=1 & \Rightarrow<\left(\mathbf{x}_{\mathbf{p}_{i}}(\tau)-\mathbf{s}_{\mathbf{p}_{j}}(\tau)\right), \mathbf{v}_{q}>\leq R \\
& \text { for all } q=\left\{1, \ldots, n_{d}\right\}
\end{aligned}
$$

a binary indicator variable for target interception at a single time instant $\tau$ within the planning horizon. The logical relation (17) can be represented by

$$
<\left(\mathbf{x}_{\mathbf{p}_{i}}(\tau)-\mathbf{s}_{\mathbf{p}_{j}}(\tau)\right), \mathbf{v}_{q}>-R \leq M_{b i g}\left(1-b_{i j}(\tau)\right),
$$

where $M_{b i g}$ is the big-M constant commonly employed in mixed-integer programming [19]. Here any $M_{b i g} \geq \operatorname{diam}(G)-R$ is an acceptable value for $M_{b i g}$. 
We now define the variables $\delta_{i}(\tau) \in\{0,1\}$ as

$$
\delta_{i}(\tau)= \begin{cases}1 & \text { if target } i \text { intercepted at } \tau_{\text {int }} \leq \tau \\ 0 & \text { if target } i \text { not intercepted for any } \tau_{\text {int }} \leq \tau\end{cases}
$$

for any $\tau_{\text {int }} \in[t, \tau]$. Equations (17) and (19) imply

$$
\sum_{\tau_{\text {int }}=t}^{\tau} \sum_{j=1}^{M} b_{i j}\left(\tau_{\text {int }}\right) \geq 1 \Leftrightarrow \delta_{i}(\tau)=1
$$

The logical relation (20) can be equivalently written in terms of linear inequalities [19] as,

$$
\begin{aligned}
& 1-\sum_{\tau_{\text {int }}=t}^{\tau} \sum_{j=1}^{M} b_{i j}\left(\tau_{\text {int }}\right) \leq\left(1-\delta_{i}(\tau)\right) \\
& 1-\sum_{\tau_{\text {int }}=t}^{\tau} \sum_{j=1}^{M} b_{i j}\left(\tau_{\text {int }}\right) \geq \epsilon+\left(1-(\tau+1) M_{b i g}-\epsilon\right) \delta_{i}(\tau),
\end{aligned}
$$

where $\epsilon>0$ is a small positive number that can be typically set to machine precision for practical implementation. The binary indicator variables $\delta_{i}(\tau)$ and (21) cause target $i$ to be considered intercepted for the remainder of the optimization horizon, and thus its interception contribute to the cost function minimization even if the sensor moves away from target $i$ in order to intercept other targets.

As with interception, we desire a binary representation of target threat for use within our MILP framework. Let $\kappa_{i}(\tau)$ be the binary indicators that represent if target $i$ is in area $A$ at time $\tau$. Additionally, we define binary indicators $\gamma_{i}(\tau)$ for target $i$ having a trajectory that will enter the area $A$ up 
to time $\tau+T^{\prime}$ in the future. Thus,

$$
\gamma_{i}(\tau)= \begin{cases}1 & \text { if } \mathbf{x}_{\mathbf{p}_{i}}\left(\tau^{\prime}\right) \in A \text { for any } \tau^{\prime} \in\left[\tau, \tau+T^{\prime}\right] \\ 0 & \text { if } \mathbf{x}_{\mathbf{p}_{i}}\left(\tau^{\prime}\right) \notin A \text { for all } \tau^{\prime} \in\left[\tau, \tau+T^{\prime}\right]\end{cases}
$$

Note that since the target motion is assumed to be independent of agent dynamics, the values of $\kappa_{i}(\tau)$ and $\gamma_{i}(\tau)$ may be calculated outside the MILP optimization process, and thus do not require linear approximations. If one desires similar linear approximations as $\delta_{i}(\tau)$ and $b_{i j}(\tau)$, we refer to [20].

Since $\mathbf{x}_{\mathbf{p}_{i}}(\tau)$ and $\mathbf{s}_{\mathbf{p}_{j}}(\tau)$ are random vectors, from (18) and (21), the binary variables $b_{i j}(\tau), \delta_{i}(\tau)$ as well as $\kappa_{i}\left(\tau^{\prime}\right)$, and $\gamma_{i}(\tau)$ are random variables as well. However the distribution of these binary random variables cannot be expressed in closed form, and thus a particle approximation is required.

\subsection{Particle Approximation of Indicator Variables for Event Probabilities}

We now discuss a particle approximation of the stochastic variables used in our motion control framework. We first develop a general methodology for creating particle-based indicator variables that may be used in the development of the probabilities required for Bayes risk, and then turn to the specific definitions used in the target interception problem.

A particle-based characterization of probabilities used in Bayes risk-based motion control such as in the target interception problem, requires a probability distribution from which we can sample the random variables involved. For the multi-agent motion control framework we describe in section 2.3, we consider a time horizon of $T$ with unit increments from an initial time $t$. In this case the random variables involved are $\mathbf{x}_{i}(t), \mathbf{s}_{j}(t), \boldsymbol{\nu}_{i}\left(t: t+T+T^{\prime}-1\right)$ and $\boldsymbol{\mu}_{j}(t: t+T-1)$ for target $i \in\{1, \ldots, N\}$ and vehicle $j \in\{1, \ldots, M\}$, 
respectively. For the case of target interception, where all target states $\mathbf{x}_{i}(t)$ are probabilistically independent, we can define a vector

$$
\mathbf{z}=\left[\begin{array}{c}
\left\{\mathbf{x}_{i}(t)\right\}_{i=1}^{N} \\
\left\{\mathbf{s}_{j}(t)\right\}_{j=1}^{M} \\
\left\{\boldsymbol{\nu}_{i}\left(t: t+T^{\prime}+T-1\right)\right\}_{i=1}^{N} \\
\left\{\boldsymbol{\mu}_{j}(t: t+T-1)\right\}_{j=1}^{M}
\end{array}\right]
$$

to contain all the random variables needed to describe all the trajectories until the time horizon $T$. In order to generate target and agent vehicle sample trajectories we need to incorporate samples of $\mathbf{z}$ and values of the vehicle control variables $\mathbf{u}_{j}(\tau)$ in $(12)$. The prediction is created by by forwardprojecting a set of $L$ particles at each step within the planning interval as the sample

$$
\mathbf{s}_{j l}(\tau+1)=\mathbf{g}_{\mathbf{j}}\left(\mathbf{s}_{j l}(\tau), \mathbf{u}_{j}(\tau), \mu_{j l}(\tau)\right)
$$

where $l \in L$. We develop particle approximations to the binary variables from (17) and (22) in terms of a generic event $D$. For a binary indicator function $I_{D}\left(\mathbf{x}\left(t: t+T+T^{\prime}\right),\left\{\mathbf{s}_{j}(t: t+T)\right\}_{j=1}^{M}\right)$ of event $D$, we have,

$$
\begin{aligned}
& I_{D}\left(\mathbf{x}(t: t+T),\left\{\mathbf{s}_{j}(t: t+T)\right\}_{j=1}^{M}\right)= \\
& \begin{cases}1 & \text { If }\left\{\mathbf{x}\left(t: t+T+T^{\prime}\right),\left\{\mathbf{s}_{j}(t: t+T)\right\}_{j=1}^{M}\right\} \in D \\
0 & \text { If }\left\{\mathbf{x}(t: t+T),\left\{\mathbf{s}_{j}(t: t+T)\right\}_{j=1}^{M}\right\} \notin D .\end{cases}
\end{aligned}
$$

As the probability of an event $D$ is given by

$$
P(D)=E\left[I_{D}\left(\left\{\mathbf{x}\left(t: t+T+T^{\prime}\right)_{i=1}^{N}\right\},\left\{\mathbf{s}_{j}(t: t+T)_{j=1}^{M}\right\}\right)\right]
$$


its finite sample approximation is given by

$$
P(D) \approx \frac{1}{L} \sum_{l=1}^{L} w_{l} I_{D}\left(\left\{\mathbf{x}_{l}\left(t: t+T+T^{\prime}\right)\right\},\left\{\mathbf{s}_{j l}(t: t+T)_{j=1}^{M}\right\}\right)
$$

where $w_{l}$ is a sample weight. Equations (24)-(27) outline an approximation to the probabilities using a particle approach. However, we refer to the reader [21] for more details. The samples $\mathbf{z}_{l}$, for $l=1, \ldots, L$ can be generated using a proposal density $q_{\mathbf{z}}(\mathbf{z})$ which can in general be different from the true density $p_{\mathbf{z}}(\mathbf{z})$.

The corresponding target and agent sample trajectories can be generated using the samples $\mathbf{z}_{l}$, as well as particle-based agent trajectories (24) and target trajectories

$$
\mathbf{x}_{i l}(\tau+1)=\mathbf{f}_{\mathbf{i}}\left(\mathbf{x}_{i l}(\tau), \nu_{i l}(\tau)\right)
$$

that are directly obtained from (12) and (10), respectively. We can then use the target and agent sample trajectories $\left\{\mathbf{x}_{i l}(t: t+T)\right\}_{i=1}^{N}$ and $\left\{\mathbf{s}_{j l}(t:\right.$ $t+T)\}_{j=1}^{M}$ to compute the expected values of various functions of the state variables using the relation,

$$
\begin{aligned}
& E\left[g \left(\left\{\mathbf{x}_{i}\left(t: t+T+T^{\prime}\right)\right\}_{i=1}^{N},\right.\right. \\
& \left.\left.\quad\left\{\mathbf{s}_{j}(t: t+T)\right\}_{j=1}^{M}\right)\right]=\lim _{L \rightarrow \infty} \frac{1}{L} \sum_{l=1}^{L} w_{l} g_{l}
\end{aligned}
$$


where,

$$
\begin{aligned}
& w_{l}=\frac{p_{\mathbf{z}}\left(\mathbf{z}_{l}\right)}{q_{\mathbf{z}}\left(\mathbf{z}_{l}\right)} \\
& g_{l}=g\left(\left\{\mathbf{x}_{i l}\left(t: t+T+T^{\prime}\right)_{i=1}^{N}\right\},\left\{\mathbf{s}_{j l}(t: t+T)_{j=1}^{M}\right\}\right) .
\end{aligned}
$$

and $g(\cdot)$ is a general relation between $\left\{\mathbf{x}_{i l}\left(t: t+T+T^{\prime}\right)_{i=1}^{N}\right\}$ and $\left\{\mathbf{s}_{j l}(t:\right.$ $\left.t+T)_{j=1}^{M}\right\}$. In (31), $w_{l}$ is the sample weight for sample $l$ and $g_{l}$ is the function evaluated at the $l^{\text {th }}$ sample trajectory. For a finite $L$ we have,

$$
\begin{aligned}
& E\left[g \left(\left\{\mathbf{x}_{i}\left(t: t+T+T^{\prime}\right)\right\}_{i=1}^{N},\right.\right. \\
& \left.\left.\left\{\mathbf{s}_{j}(t: t+T)\right\}_{j=1}^{M}\right)\right] \approx \frac{1}{L} \sum_{l=1}^{L} w_{l} g_{l} .
\end{aligned}
$$

\subsection{Particle-based Bayes Risk for Target Interception}

We now turn to defining the particle-based events of threat and interception using the general particle approximation in $(27)$. We define $b_{i j l}(\tau)$, $\delta_{i l}(\tau), \kappa_{i l}\left(\tau^{\prime}\right)$ and $\gamma_{i l}(\tau)$ for $\tau \in[t, t+T]$ and $\tau^{\prime} \in\left[\tau, \tau+T^{\prime}\right]$ to be the particle realizations of the indicator variables $b_{i j}(\tau), \delta_{i}(\tau), \kappa_{i}(\tau)$ and $\gamma_{i}(\tau)$ defined in (18) and (21). Thus rewriting (18) and (21) in terms of the particle tra-

jectory $l \in\{1, \ldots, L\}$ we get the following relations defining $b_{i j l}(\tau), \delta_{i l}(\tau)$, $\kappa_{i l}\left(\tau^{\prime}\right)$ and $\gamma_{i l}(\tau)$ :

$$
<\left(\mathbf{x}_{\mathbf{p}_{i l}}(\tau)-\mathbf{s}_{\mathbf{p}_{j l}}(\tau)\right), \mathbf{v}_{q}>-R \leq M_{b i g}\left(1-b_{i j l}(\tau)\right)
$$




$$
\begin{aligned}
& 1-\sum_{\tau^{\prime}=t}^{t+\tau} \sum_{j=1}^{M} b_{i j l}\left(\tau^{\prime}\right) \leq\left(1-\delta_{i l}(\tau)\right) \\
& 1-\sum_{\tau^{\prime}=t}^{t+\tau} \sum_{j=1}^{M} b_{i j l}\left(\tau^{\prime}\right) \geq \epsilon+(1-(\tau+1) M-\epsilon) \delta_{i l}(\tau),
\end{aligned}
$$

We define $\left(\Gamma_{i}^{\text {past }}\right)$ as the event that the target has been intercepted at $t_{i}<t$. Likewise, $\sim \Gamma_{i}^{\text {past }}$ is the event that the target was not intercepted before time $t_{i}<t$. Let $E_{i}(\tau)$ be the event that a target's trajectory enters the region $A$ within the time horizon $\left[\tau, \tau+T^{\prime}\right]$. Then the conjunctive probability that target $i$ is a threat at time $\tau$ is

$$
P\left(\Omega_{i}(\tau)\right)=P\left(E_{i}(\tau) \cap \sim \Gamma_{i}^{\text {past }}\right)
$$

Using the particle-based definitions found in (33)-(35) for calculating the probabilities of threat and interception first defined in (6) and (8) yields

$$
P\left(\Omega_{i}(\tau)\right) \approx \tilde{P}\left(\Omega_{i}(\tau)\right)=\frac{1}{L} \sum_{l=1}^{L} w_{l} \gamma_{i l}(\tau)\left(\sim \Gamma_{i}^{\text {past }}\right)
$$

and

$$
P\left(\Gamma_{i}(\tau)\right) \approx \tilde{P}(\Gamma(\tau))=\frac{1}{L} \sum_{l=1}^{L} w_{l} \delta_{i l}(\tau) .
$$

We can now use the particle filter approximations for the probabilities in 
(36) and (37) to approximate the Bayes risk for each individual target as

$$
\begin{aligned}
\tilde{R}_{i}(\tau) & =C_{0,1}\left(1-\tilde{P}\left(\Gamma_{i}(\tau)\right)\right) \tilde{P}\left(\Omega_{i}(\tau)\right) \\
& +C_{1,1} \tilde{P}\left(\Gamma_{i}(\tau)\right) \tilde{P}\left(\Omega_{i}(\tau)\right) \\
& +C_{0,0}\left(1-\tilde{P}\left(\Gamma_{i}(\tau)\right)\right)\left(1-\tilde{P}\left(\Omega_{i}(\tau)\right)\right) \\
& +C_{1,0} \tilde{P}\left(\Gamma_{i}(\tau)\right)\left(1-\tilde{P}\left(\Omega_{i}(\tau)\right)\right) .
\end{aligned}
$$

Summing (38) over all targets over the entire optimization interval $[t, t+T]$, we have our final cost function for Bayes risk in a receding horizon framework,

$$
R_{R H C}=\sum_{\tau=t}^{t+T} \sum_{i}^{N} \tilde{R}_{i}(\tau) .
$$

Using constraints (33)-(34), (36), (37), and our receding horizon policy (39), the following summarized optimization problem is the MILP framework 
corresponding to the area protection.

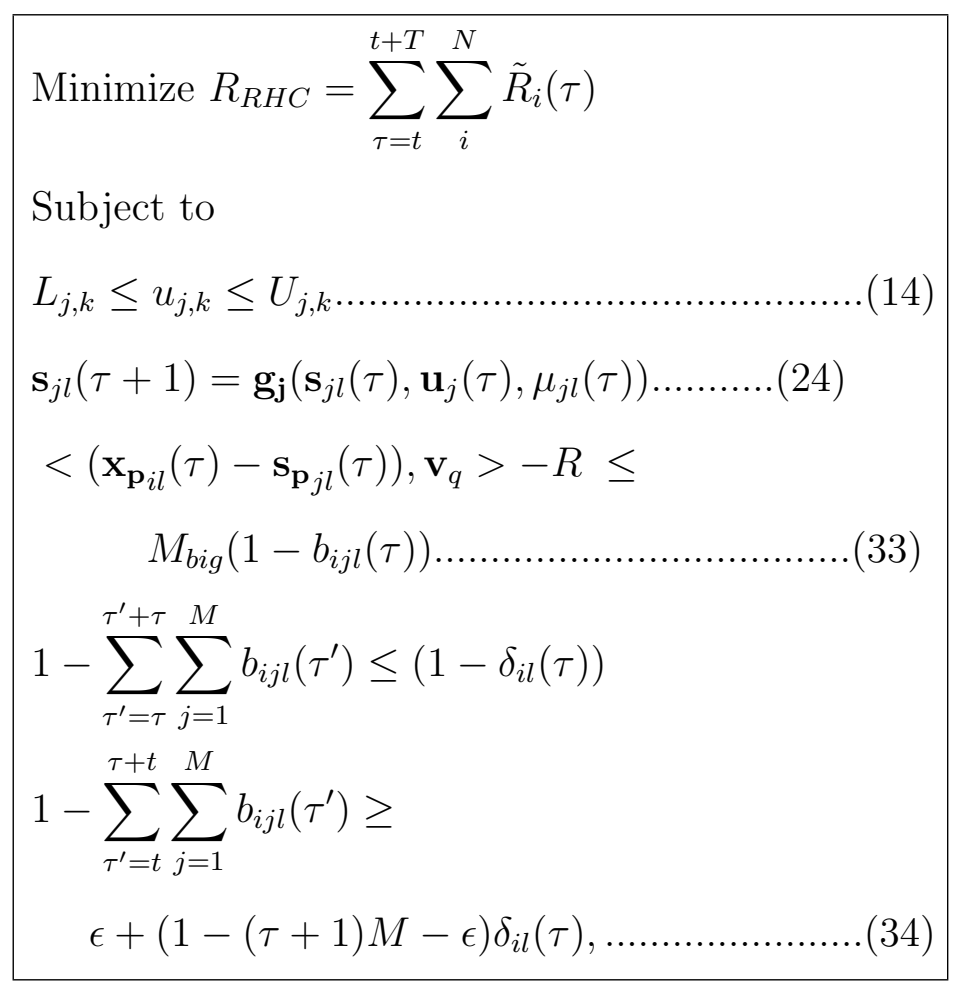

In (40), the primary optimization variables are the entire sequence of control actions for the optimization interval $[t, t+T]$, namely $\left\{\left\{\mathbf{u}_{j}(\tau)\right\}_{j=1}^{M}\right\}_{\tau=t}^{t+T}$. As the particles of the agent trajectory $\left\{\left\{\left\{\mathbf{s}_{j l}(\tau)\right\}_{j=1}^{M}\right\}_{\tau=t}^{t+T}\right\}_{l=1}^{L}$ depend on the agent control inputs, they too are the variables of the optimization problem. The samples $\left\{\beta_{l}\right\}_{l=1}^{L}$ are dependent on the agent trajectories, and thus are functions of the optimization problem as well.

We have thus far described a method for using a cost function derived from Bayes risk for motion control in a purely target interception-centric manner. We now turn to extending our Bayes risk motion control framework to incorporating collision avoidance. 


\section{MINIMUM-RISK COLLISION AVOIDANCE}

Inspired by the use of multiple stages for a collision alert system in [22], we develop the collision event and corresponding mitigation procedure in a twostage manner. The benefit of multiple stages in a collision event allows for the relaxation of hard constraints in order to achieve other objectives while maintaining a desired level of safety. In the first stage of this methodology, the agents must decide whether a near-miss event will occur. A near-miss is the event where two agents violate a user-specified radius between each other $R_{c}$. The decision of an occurrence or non-occurrence of a near-miss between agents $j$ and $j^{\prime}$ at time $\tau \in[t, t+T]$ is denoted by $\Psi_{j j^{\prime}}(\tau)$ or $\sim \Psi_{j j^{\prime}}(\tau)$, respectively. The occurrence of a near-miss can be written formally as

$$
\left.\| \mathbf{s}_{\mathbf{p}_{j}}(\tau)-\mathbf{s}_{\mathbf{p}_{j^{\prime}}}(\tau)\right) \| \leq R_{c}
$$

In the second stage, we define the events of collision and non-collision as an event that is damaging to one or both agents. These classes are denoted $\mathcal{C}_{j j^{\prime}}(\tau)$ and $\sim \mathcal{C}_{j j^{\prime}}(\tau)$, respectively. Due to agent shapes being typically nonconvex and the possibility of collisions occurring in between optimization steps, among other concerns, it is appropriate to define the probabilities of events $\mathcal{C}_{j j^{\prime}}(\tau)$ and $\sim \mathcal{C}_{j j^{\prime}}(\tau)$ in terms of probabilities based on violations of the near-miss radius rather than physical properties of the agents themselves. 
As such, we define the conditional collision priors

$$
\begin{aligned}
P\left(\mathcal{C}_{j j^{\prime}}(\tau) \mid \Psi_{j j^{\prime}}(\tau)\right) & =P_{c} \\
P\left(\mathcal{C}_{j j^{\prime}}(\tau) \mid \sim \Psi_{j j^{\prime}}(\tau)\right) & =0 \\
P\left(\sim \mathcal{C}_{j j^{\prime}}(\tau) \mid \Psi_{j j^{\prime}}(\tau)\right) & =1-P_{c} \\
P\left(\sim \mathcal{C}_{j j^{\prime}}(\tau) \mid \sim \Psi_{j j^{\prime}}(\tau)\right) & =1 .
\end{aligned}
$$

Thus, when the motion of two agents cause a violation of the near-miss radii, there is a pre-defined probability of a collision occurring. When the motion of two agents do not violate their near-miss radii, there is no chance of a collision occurring.

We now wish to form a cost function for risk of collision similar to Bayes risk from (3) for this scenario. We first note that in the standard definition of Bayes risk, the hypothesis is conditioned on the actual event, as written in (4). However, in (42), the events are reversed. In order to write the Bayes risk in the proper form, we use Bayes' rule to define the near-miss hypothesis 
conditioned on a collision occurring as

$$
\begin{gathered}
P\left(\Psi_{j j^{\prime}}(\tau) \mid \mathcal{C}_{j j^{\prime}}(\tau)\right)= \\
\frac{P\left(\mathcal{C}_{j j^{\prime}}(\tau) \mid \Psi_{j j^{\prime}}(\tau)\right) P\left(\Psi_{j j^{\prime}}(\tau)\right)}{P\left(\mathcal{C}_{j j^{\prime}}(\tau)\right)} \\
P\left(\sim \Psi_{j j^{\prime}}(\tau) \mid \mathcal{C}_{j j^{\prime}}(\tau)\right)= \\
\frac{P\left(\mathcal{C}_{j j^{\prime}}(\tau) \mid \sim \Psi_{j j^{\prime}}(\tau)\right) P\left(\sim \Psi_{j j^{\prime}}(\tau)\right)}{P\left(\mathcal{C}_{j j^{\prime}}(\tau)\right)} \\
P\left(\Psi_{j j^{\prime}}(\tau) \mid \sim \mathcal{C}_{j j^{\prime}}(\tau)\right)= \\
\frac{P\left(\sim \mathcal{C}_{j j^{\prime}}(\tau) \mid \Psi_{j j^{\prime}}(\tau)\right) P\left(\Psi_{j j^{\prime}}(\tau)\right)}{P\left(\sim \mathcal{C}_{j j^{\prime}}(\tau)\right)} \\
P\left(\sim \Psi_{j j^{\prime}}(\tau) \mid \sim \mathcal{C}_{j j^{\prime}}(\tau)\right)= \\
\frac{P\left(\sim \mathcal{C}_{j j^{\prime}}(\tau) \mid \sim \Psi_{j j^{\prime}}(\tau)\right) P\left(\sim \Psi_{j j^{\prime}}(\tau)\right)}{P\left(\sim \mathcal{C}_{j j^{\prime}}(\tau)\right)},
\end{gathered}
$$

where $P\left(\Psi_{j j^{\prime}}(\tau)\right)$ and $P\left(\mathcal{C}_{j j^{\prime}}(\tau)\right)$ are the corresponding prior probabilities of a near-miss and collision, respectively.

We can now write the prior-averaged conditional risks associated with the event of non-collision and collision as

$$
\begin{aligned}
R_{\sim \mathcal{C}_{j j^{\prime}}}(\tau)= & C_{0,0}^{\prime} P\left(\sim \Psi_{j j^{\prime}}(\tau) \mid \sim \mathcal{C}_{j j^{\prime}}(\tau)\right) \\
& +C_{1,0}^{\prime} P\left(\Psi_{j j^{\prime}}(\tau) \mid \sim \mathcal{C}_{j j^{\prime}}(\tau)\right) \\
R_{\mathcal{C}_{j j^{\prime}}}(\tau)= & C_{0,1}^{\prime} P\left(\sim \Psi_{j j^{\prime}}(\tau) \mid \mathcal{C}_{j j^{\prime}}(\tau)\right) \\
& +C_{1,1}^{\prime} P\left(\Psi_{j j^{\prime}}(\tau) \mid \mathcal{C}_{j j^{\prime}}(\tau)\right)
\end{aligned}
$$


and collision risk as

$$
R_{j j^{\prime}}(\tau)=R_{\mathcal{C}_{j j^{\prime}}}(\tau) P\left(\mathcal{C}_{j j^{\prime}}(\tau)\right)+R_{\sim \mathcal{C}_{j j^{\prime}}}(\tau) P\left(\sim \mathcal{C}_{j j^{\prime}}(\tau)\right)
$$

Substituting the probabilities from (43)-(46) into the conditional risks (47), and (47) into (48), we have the risk for a collision based on the hypothesis of violating a near-miss radius between agents $j$ and $j^{\prime}$, written

$$
\begin{aligned}
R_{j j^{\prime}}(\tau)= & C_{1,0}^{\prime} P\left(\sim \mathcal{C}_{j j^{\prime}}(\tau) \mid \Psi_{j j^{\prime}}(\tau)\right) P\left(\Psi_{j j^{\prime}}(\tau)\right) \\
& +C_{0,0}^{\prime} P\left(\sim \mathcal{C}_{j j^{\prime}}(\tau) \mid \sim \Psi_{j j^{\prime}}(\tau)\right) P\left(\sim \Psi_{j j^{\prime}}(\tau)\right) \\
& +C_{1,1}^{\prime} P\left(\mathcal{C}_{j j^{\prime}}(\tau) \mid \Psi_{j j^{\prime}}\right) P\left(\Psi_{j j^{\prime}(\tau)}\right) \\
& +C_{0,1}^{\prime} P\left(\mathcal{C}_{j j^{\prime}}(\tau) \mid \sim \Psi_{j j^{\prime}}(\tau)\right) P\left(\sim \Psi_{j j^{\prime}}(\tau)\right) \\
= & C_{1,0}^{\prime}\left(1-P_{c}\right) P\left(\Psi_{j j^{\prime}}(\tau)\right)+C_{0,0}^{\prime} P\left(\sim \Psi_{j j^{\prime}}(\tau)\right) \\
& +C_{1,1}^{\prime} P_{c} P\left(\Psi_{j j^{\prime}}(\tau)\right) .
\end{aligned}
$$

While typical Bayes risk is used to optimize decision rules, our risk function derived herein departs from the typical use by penalizing incorrect decisions as well as a correct decision that a collision will occur. However, because this risk function is derived from the definition of Bayes risk, it's form is comparable. Thus, combining the risk of collision with the previously derived Bayes risk for interception is an appropriate cost function for balancing the risk of collision with the risk of incorrect classification and interception of incoming targets.

Next, we develop a particle approximation for the probability of a near- 
miss event. We first create the linear approximation for (41) as

$$
<\left(\mathbf{s}_{\mathbf{p}_{j}}(\tau)-\mathbf{s}_{\mathbf{p}_{j^{\prime}}}(\tau)\right), \mathbf{v}_{q}>\leq R_{c} \text { for all } q=\left\{1, \ldots, n_{d}\right\}
$$

We then use the binary variable $c_{j j^{\prime} l}(\tau)$ to indicate violation of near-miss radius between agents $j$ and $j^{\prime}$ at time $t$. Thus, we have the constraint

$$
\begin{array}{r}
<\left(\mathbf{s}_{\mathbf{p}_{j}}(\tau)-\mathbf{s}_{\mathbf{p}_{j}^{\prime}}(\tau)\right), \mathbf{v}_{q}>\leq R_{c} \Rightarrow c_{j j^{\prime} l}(\tau)=1 \\
\text { for all } q=\left\{1, \ldots, n_{d}\right\}
\end{array}
$$

This logical relation can be represented with a set of inequalities and the binary variable $g_{j j^{\prime} l q}(\tau)$,

$$
\begin{aligned}
<\left(\mathbf{s}_{\mathbf{p}_{j l}}(\tau)-\mathbf{s}_{\mathbf{p}_{j^{\prime} l}}(\tau)\right), \mathbf{v}_{q} & >-R_{c} \\
& \geq \epsilon+\left(-M_{b i g}-\epsilon\right) g_{j j^{\prime} l q}(\tau) \\
-\sum_{q=1}^{n_{d}} g_{j j^{\prime} l q}(\tau)+n_{q} & \geq \epsilon+\left(-M_{b i g}-\epsilon\right) c_{j j^{\prime} l}(\tau)
\end{aligned}
$$

using big-M notation. The particle-based approximation to the probability of a near-miss can be written as

$$
P\left(\Psi_{j j^{\prime}}(\tau)\right) \approx \tilde{P}\left(\Psi_{j j^{\prime}}(\tau)\right)=\frac{1}{L} \sum_{l=1}^{L} w_{l} c_{j j^{\prime} l}(\tau)
$$

with corresponding complement

$$
\tilde{P}\left(\sim \Psi_{j j^{\prime}}(\tau)\right)=1-\tilde{P}\left(\Psi_{j j^{\prime}}(\tau)\right) .
$$


The particle-based risk for collision avoidance can now be written as

$$
\begin{aligned}
\tilde{R}_{j j^{\prime}}(\tau)= & C_{1,0}^{\prime}\left(1-P_{c}\right) \tilde{P}\left(\Psi_{j j^{\prime}}(\tau)\right)+C_{0,0}^{\prime} \tilde{P}\left(\sim \Psi_{j j^{\prime}}(\tau)\right) \\
& +C_{1,1}^{\prime} P_{c} \tilde{P}\left(\Psi_{j j^{\prime}}(\tau)\right)
\end{aligned}
$$

We now sum (55) across all permutations of agents $j$ and $j^{\prime}$ to find the total Bayes risk for collision avoidance,

$$
\tilde{R}_{c o l}(\tau)=\sum_{j=1}^{M} \sum_{j^{\prime}=1, j^{\prime} \neq j}^{M} \tilde{R}_{j j}(\tau)
$$

Finally, we add (56) to the summation in (39) to obtain total risk across the time horizon at time $t$,

$$
\tilde{R}_{R H C}^{\prime}=\sum_{\tau=t}^{t+T}\left[\sum_{i=1}^{N} \tilde{R}_{i}(\tau)+R_{c o l}(\tau)\right] .
$$

We note that if one assumes that the costs $C_{0,0}^{\prime}=C_{1,0}^{\prime}=0$ in all circumstances, one can arrive at an objective function to minimize the expected cost of collision by simply summing $C_{1,1}^{\prime} P\left(\Psi_{j j^{\prime}}(\tau)\right)$ over all agent pairs, as the constant $P_{c}$ would then drop out. However, formulating the collision problem in our Bayes risk framework using (43)-(49) has two primary benefits. First, explicitly deriving the cost in a manner similar to Bayes risk allows for every event combination of $\mathcal{C}_{j j^{\prime}}(\tau), \sim \mathcal{C}_{j j^{\prime}}(\tau), \Psi_{j j^{\prime}}(\tau)$, and $\sim \Psi_{j j^{\prime}}(\tau)$, to be factored into a prior-averaged conditional risk and minimized in the objective function. While simply penalizing the collision cost may be appropriate in some scenarios such as automotive collision avoidance, collision avoidance in other 
scenarios such as airspace management for large aircraft treat near-misses as problematic as well (albeit to a less extent than an actual collision), leading to after-incident reviews and recommendations for prevention [23]. Using the broader definition of Bayes risk allows for prior-averaged estimated costs for all event combinations to be factored into the objective function. Secondly, the desire for obstacle avoidance behaviors does not exist in a vacuum. Obstacle avoidance is a secondary albeit critical objective that must be balanced with the primary tasking. Deriving collision avoidance formally within the Bayes risk setting introduced previously allows it to be rigorously included with target interception. The result is a formally correct way to assign the relative costs and compute the average risk of events related to both collision and interception.

\section{NUMERICAL IMPLEMENTATION}

Now that the theoretical framework for the stochastic receding-horizon MILP problem has been discussed, we will now outline our specific implementations used in simulations, as well as provide a discussion on the additional steps and considerations that may be necessary for implementation on actual systems. As discussed previously, $t$ represents the current timestep, with receding-horizon time interval $[t, t+T]$. We have the state equations (10) 
and (12) of the system in our implementation as

$$
\begin{aligned}
\mathbf{x}_{i}(t+1) & =\left[\begin{array}{llll}
1 & 0 & 1 & 0 \\
0 & 1 & 0 & 1 \\
0 & 0 & 1 & 0 \\
0 & 0 & 0 & 1
\end{array}\right] \mathbf{x}_{i}(t)+\boldsymbol{\nu}_{i}(t) \\
\mathbf{s}_{j}(t+1) & =\left[\begin{array}{ll}
1 & 0 \\
0 & 1
\end{array}\right] \mathbf{s}_{j}(t)+\mathbf{u}_{j}(t)+\boldsymbol{\mu}_{j}(t) .
\end{aligned}
$$

For the agent model represented by (59) we have $\left(\mathbf{s}_{j}\right)_{p}(t)=\mathbf{s}_{j}(t)$ and hence $\mathbf{L}_{\text {sen }}=\mathbf{I}_{2 \times 2}$. We assume the agents can measure the relative target position as

$$
\mathbf{y}_{i j}(t)=\mathbf{x}_{\mathbf{p}_{i}}(t)-\mathbf{s}_{\mathbf{p}_{j}}(t)+\boldsymbol{\eta}_{i j}(t) .
$$

In addition to the cost shown in (39), we have included a small travel cost for each vehicle, making the total RHC component

$$
J_{R H C}=R_{R H C}+\sum_{\tau=t}^{t+T} \sum_{j=1}^{M} c_{s} f_{j}(\tau)
$$

where $c_{s}=1 \times 10^{-5}$, and $f_{j}(\tau)$ is a linear approximation for the speed of vehicle $j$, given by

$$
<\mathbf{u}_{j}\left(t^{\prime}\right), \mathbf{v}_{q}>\leq f_{j}(\tau) \text { for all } q=\left\{1, \ldots, n_{d}\right\}
$$

The addition of travel cost to the Bayes risk cost ensures that minimizing 
the cost function will result in reasonably short paths for the same target interception result.

\subsection{Kalman Filter Estimation}

While the theoretical framework discussed in the previous sections can be used for any stochastic noise model, as a test case we first present am implementation where the system and measurement noise of the target and agents to be zero mean Gaussian. For the process noise of the targets and agents, we assume

$$
\begin{aligned}
E\left[\boldsymbol{\nu}_{i}(t)\left(\boldsymbol{\nu}_{i}(t)\right)^{\top}\right] & =\mathbf{Q}_{i} \\
E\left[\boldsymbol{\mu}_{j}(t)\left(\boldsymbol{\mu}_{j}(t)\right)^{\top}\right] & =\mathbf{S}_{j} .
\end{aligned}
$$

For the measurement noise, we assume

$$
E\left[\boldsymbol{\eta}_{i j}(t)\left(\boldsymbol{\eta}_{i j}(t)\right)^{\top}\right]=\mathbf{R}_{i j}
$$

Additionally we assume the PDFs of the target and the agent states at $t$ of the RHC planning interval to be Gaussian. These PDFs can be estimated by incorporating all the previous observations using a Kalman filter due to the assumed linear Gaussian system. Let the state for agent $j$ at $t=0$ be distributed as $\mathbf{s}_{j}(t) \sim N\left(\hat{\mathbf{s}}_{j}(t), \mathbf{P}_{j}(t)\right)$.

A key feature of our implementation is the efficient computation of the control by significantly reducing the number of optimization variables in the MILP given in (40). The minimal number of variables is possible for the agent dynamics given by (59) due to the additive noise assumption. With 
the proposed method we can represent the agent state particles $\mathbf{s}_{j l}(t)$ in terms of the agent state predictions $\hat{\mathbf{s}}_{j}(t)$ and the sampled random parameters. The propagation of particles is performed as follows:

The agent state particles corresponding to (59) propagate as

$$
\mathbf{s}_{j l}(t+1)=\mathbf{s}_{j l}(t)+\mathbf{u}_{j}(t)+\boldsymbol{\mu}_{j l}(t) .
$$

Because optimization typically occurs over individual control actions $\mathbf{u}_{j}(t)$ for each agents, we may optimize over each agent's distribution $\hat{\mathbf{s}}_{j}(t+1)$. Therefore the agent state prediction due to zero mean $\boldsymbol{\mu}_{j}(t)$ propagates as,

$$
\hat{\mathbf{s}}_{j}(t+1)=\hat{\mathbf{s}}_{j}(t)+\mathbf{u}_{j}(t)
$$

The noise in the initial conditions,

$$
\boldsymbol{\mu}_{j}^{i n i}=\mathbf{s}_{j}(t)-\hat{\mathbf{s}}_{j}(t)
$$

is distributed as $\boldsymbol{\mu}_{j}^{i n i} \sim N\left(\mathbf{0}, \mathbf{P}_{j}(t)\right)$. Particle $l$ of the initial state is given by,

$$
\mathbf{s}_{j l}(t)=\hat{\mathbf{s}}_{j}(t)+\boldsymbol{\mu}_{j l}^{i n i}
$$

By recursively updating agent particle state (68) using (65) and by using the relation (66) we get,

$$
\mathbf{s}_{j l}(t)=\hat{\mathbf{s}}_{j}(t)+\zeta_{j l}(t)
$$

where,

$$
\boldsymbol{\zeta}_{j l}(t)=\boldsymbol{\mu}_{j l}^{i n i}+\sum_{\tau=t}^{t+T-1} \boldsymbol{\mu}_{j l}(\tau)
$$


Thus (69) is a representation of the agent state particles in terms of the agent state prediction.

In our implementation we use (69) to replace the agent state particles $\mathbf{s}_{j l}(t)$, in terms of agent state predictions $\hat{\mathbf{s}}_{j}(t)$ and cumulative sampled disturbances $\boldsymbol{\zeta}_{j l}(t)$ at all time instants $t$ in the horizon. Using (69) in constraint (33) of the optimization program (40), constraint (33) was rewritten in terms of the agent state prediction as

$$
\begin{aligned}
<\left(\mathbf{x}_{\mathbf{p}_{i l}}(t)-\hat{\mathbf{s}}_{\mathbf{p} j}(t)\right), \mathbf{v}_{q}>-R \leq \\
M_{b i g}\left(1-b_{i j l}(t)\right)+<\boldsymbol{\zeta}_{j l}(t), \mathbf{v}_{q}>
\end{aligned}
$$

Constraint (24) which represents the evolution of the particles was hence replaced by (66), which represents the evolution of the agent state prediction. Thus in (40) $L \times T \times M$ agent state particle optimization variables were reduced to $T \times M$ agent state prediction optimization variables. While we do not explicitly compare the performance of comparison of (71) to the particle representation of agent position $\mathbf{s}_{j l}(t)$ for the same optimization problem, in generall integer variables can potentially increase the computational complexity of a MILP if the formulations are not tight, and thus limiting their use can be potentially of benefit [24].

\subsection{Particle Filter Estimation}

We now present a particle filter implementation for use with non-Gaussian noise. We assume the same system model as (58-62), except in the following case the noise components are assumed Cauchy with locational parameters equal to zero. The Cauchy distribution is a heavy-tailed, stable distribution 
with $n$-dimensional multivariate probability density function

$$
f(\mathbf{x})=\frac{\Gamma_{f}((n+1) / 2)}{\pi^{(n+1) / 2} \prod_{k=1}^{n} \gamma_{k}\left(1+\sum_{k=1}^{n} x_{k}^{2} / \gamma_{k}^{2}\right)^{(n+1) / 2}}
$$

where $x_{1}, \ldots, x_{n} \in \mathbf{x}, \gamma_{k}>0$ is a scale parameter, and $\Gamma_{f}(\cdot)$ is the gamma function [25]. In addition to providing a basic example of the viability of our optimization algorithm for use with non-Gaussian noise, it has been proposed that Cauchy random noise can provide a more accurate representation of target maneuvers when there is the possibility of an abrupt change in agent motion [26].

There are currently a variety of particle filter implementations capable of performing the required state estimation of our system. Crisan and Doucey provide a survey of many particle filter implementations and their convergence properties in [27]. We use an auxillary sample importance resampling (A-SIR) particle filter in our implementation, and briefly describe the implementation, referring to [28] for details. In this implementation, the filter generates $L$ particles of the system states, denoted $\boldsymbol{\rho}(t)$ such that

$$
\boldsymbol{\rho}(t) \sim p(\boldsymbol{\rho}(t+1) \mid \boldsymbol{\rho}(t))
$$

using the system dynamics of (58) and (59) with process noise $\boldsymbol{\nu}_{i}(t)$ and $\boldsymbol{\mu}_{j}(t)$ as Cauchy random variables. From the particles generated by (73), particle weights $\tilde{w}_{l}(t+1)$ are generated for each particle using the likelihood of the particle based on the system measurement from (60), such that

$$
\tilde{w}_{l}(t+1)=p\left(\mathbf{y}(t) \mid \boldsymbol{\rho}_{l}(t)\right) .
$$


The weights $\tilde{w}_{l}(t+1)$ are then normalized such that

$$
w_{l}(t+1)=\frac{\tilde{w}_{l}(t+1)}{\sum_{l=1}^{L} \tilde{w}_{l}(t+1)} .
$$

The particles $\boldsymbol{\rho}(t)$ and weights $w_{l}(t+1), l=1, \ldots, L$ then undergo a resampling process from [29] known as systematic resampling. These new particles are denoted $\boldsymbol{\rho}^{\prime}(t)$. Using the resampled particles, another set of particles is created such that

$$
\hat{\boldsymbol{\rho}}(t) \sim p\left(\boldsymbol{\rho}(t) \mid \boldsymbol{\rho}^{\prime}(t-1)\right)
$$

with importance weights calculated as in (75). The particles $\hat{\boldsymbol{\rho}}(t)$ are then used to generate samples for the optimization procedure as in (28) and (24).

\subsection{The Compatibility of the Framework to Real Implementation}

We now provide a brief discussion on the compatibility of our Bayes risk motion planning framework to real-world implementation. While a refined implementation of our framework that may be deployed on real systems is beyond the scope of this work, we believe the following discussion will provide guidance for its future development.

One of the primary limiting assumptions on the use of our Bayes risk motion planning framework is in the assumption of simplified linear constraints for the agent dynamics. Systems of a highly nonlinear nature may not track the motion planning trajectories accurately enough to enforce the interception assumption at a particular time step. The inability to track motion planning trajectories could then cause the probabilities and our Bayes risk-based cost function to become inaccurate as well. However, the tracking issue could be mitigated by the addition of constraints that more accurately 
approximate nonlinear dynamics, as any continuous nonlinear function can be approximated using a piecewise affine function to an arbitrary accuracy [30]. Richards and How provide a tutorial on on piecewise affine functions and their relation to control in [31]. We also note that linear assumptions on agent dynamics is a common assumption in several classes of real-world systems. Quadroters, for example, have served as testbeds for control laws developed from linear programming in several recent works such as [32, 33].

A second consideration for real implementation of our system is the use of MILP in order to perform the optimization of the motion planning framework. MILP is an NP-hard optimization process [30]. As such, problems of large numbers of (specifically integer) variables become intractable for large problems. Indeed, in our simulations, problems involving more than six agents combined with a $T=7$ planning horizon become impractical to solve in a reasonable time frame for coordinated, real-time motion control. However, for fewer agents and smaller planning horizons, we believe our framework could perform well for real-world systems. As will be discussed in the proceeding section, the average MILP solve time for $M=4$ agents and optimization interval of $T=4$ steps was 0.57 seconds. The considerably greater overall average iteration time of 8.57 seconds is due to the MILP matrices and vectors being populated within MATLAB, and not optimized for computational efficiency. If the problem setup functions were converted to a relatively fast programming language such as $\mathrm{C}++$ and populated with functions that were designed for efficiency, then performance would likely improve dramatically. Indeed, real-world implementation of various integer linear programming optimization processes have been shown in several recent 
works, even for unmanned air vehicles [34, 35].

Thirdly, the current centralized nature of our framework limits its use to systems where communication is not limited. We assume the pose of both the targets and the agents are known to a centralized planning agent, and we assume all of the agents receive their current control actions from the planner. However, the amount of data that must be communicated to either a central planner or between every agent is very small, namely the current pose of the targets and agents. While this assumption may limit the practicality of our framework in unknown or adversarial environments, protective applications such as target interception could indeed be practical.

While there are several assumptions that cause implementation of our framework to be difficult for some real-world applications, we do not believe any are so significant that the framework cannot be implemented on a real system. Each assumption has been used in real implementation of various experimental setups, and can potentially be mitigated by refinement.

\section{Simulation Results}

\subsection{Simulation Results with Kalman Filter Implementation}

We now present simulation results under the Gaussian noise assumption and Kalman filter implementation. For the cost parameters, we assume cost 
coefficients

$$
\begin{aligned}
& C_{0,0}=0 \\
& C_{1,0}=1 \\
& C_{0,1}=10 \\
& C_{1,1}=0 .
\end{aligned}
$$

The cost coefficients (77) will cause a missed-detection to be penalized highly compared to false alarms, and thus targets will be intercepted if they have a significant but small probability of being a threat.

We assume specific noise components for the state noise (63) and measurement noise (64) as

$$
\begin{aligned}
& \mathbf{Q}_{i}=\operatorname{diag}\left[\begin{array}{c}
1 \times 10^{-6} \\
1 \times 10^{-6} \\
1.6 \times 10^{-3} \\
1.6 \times 10^{-3}
\end{array}\right] \\
& \mathbf{S}_{j}=\operatorname{diag}\left[\begin{array}{ll}
4 \times 10^{-3} & 4 \times 10^{-3}
\end{array}\right] \\
& \mathbf{R}_{i j}=\operatorname{diag}\left[\begin{array}{ll}
1 \times 10^{-3} & 1 \times 10^{-3}
\end{array}\right]
\end{aligned}
$$

Figure 1 shows a simulation consisting of $M=4$ agents, $N=4$ targets, $T=4$ RHC-step, and $T^{\prime}=5$ particle time horizon at simulation time step $t=3$. The entire simulation with complete agent and target paths through simulation time step $t=10$ is shown in Figure 2. The circular radius of protection has a radius of $R_{\mathbf{p}}=2$. The magnitude of each target's velocity are 
randomly assigned $v_{m a g}^{i} \in[0.4,0.8]$, with direction towards the center of the region. The red circles represent the target starting locations. Each sensor location is overlaid with the respective simulation time-step $t$. The agents have an interception radius of $R=0.5$ under the polygonal approximation scheme. The polygonal approximation side count is $n_{d}=5$.

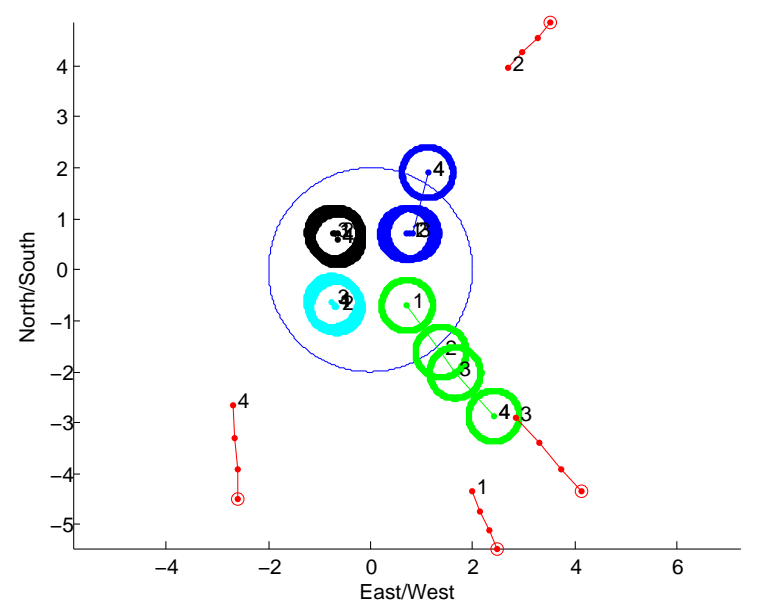

Figure 1: Simulation with $M=4, N=4, T=4, T^{\prime}=5, L=25, t=3$. Red lines are target tracks. Small circles are agent interception radii with agent paths in center. Large circle is region of protection.

As seen in Figure 2, the four agents intercept three targets that are heading into the region of protection. The simulation was run using Gurobi with a MATLAB interface on a $4 \mathrm{~GB}$ RAM, $2.4 \mathrm{GHz}$ personal computer. The average time required to run an iteration of the simulation was 8.11 seconds, with an average MILP solve time of 1.15 seconds. Maximum time to perform an iteration of the simulation was 20.0 seconds, with maximum MILP solve time of 4.3 seconds. Example particle trajectories at time step $t=3$ are shown in the Figure 3. As shown in Figure 3, although target 2 (northernmost target) has only half of the particles entering the region in the current 


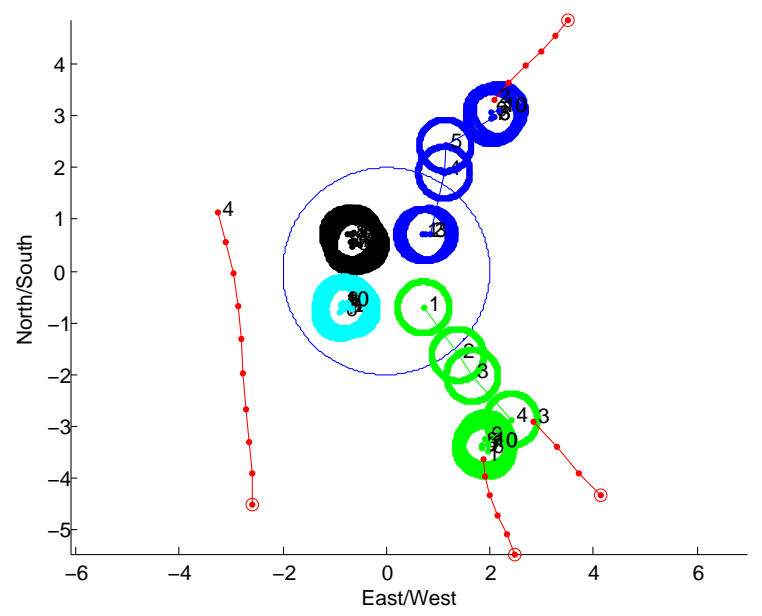

Figure 2: Simulation with $M=4, N=4, T=4, T^{\prime}=5, L=25$.

time horizon, a sensor is already committing to intercept the target due to the selected cost ratio. Target 4 (southwest target) is not being intercepted yet, as the target's threat probability is not high enough to meet the required threshold.

Figure 4 shows the minimized cumulative Bayes risk vs. time-step in the simulation. Note due to the changing proximity of the targets to the region of protection, the risk increases until the targets start to become intercepted at $t=5$. Once targets become intercepted, the Bayes risk monotonically decreases until all targets are intercepted. The Bayes risk becomes zero at timestep $t=7$ when all targets are considered intercepted.

\subsection{Simulation Results with Particle Filter Implementation}

We now present simulation results under the Cauchy noise assumption and particle filter implementation. The number of particles generated for both the particle filter estimator and the optimization algorithm were $L=45$ particles. For the cost parameters, we assume the same cost coefficients as 


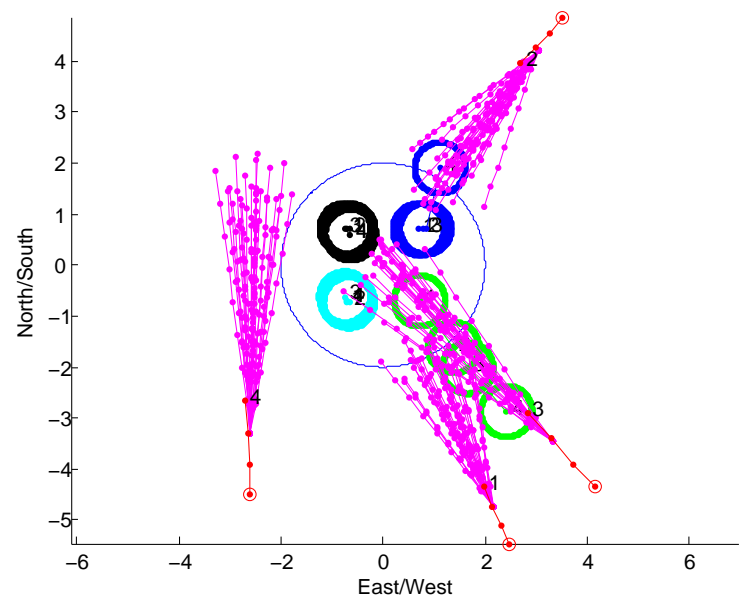

Figure 3: Simulation with $M=4, N=4, T=4, T^{\prime}=5, L=25, t=3$. Red lines are target tracks. Small circles are agent interception radii with agent paths in center. Large circle is region of protection. Purple lines are target particle projections.

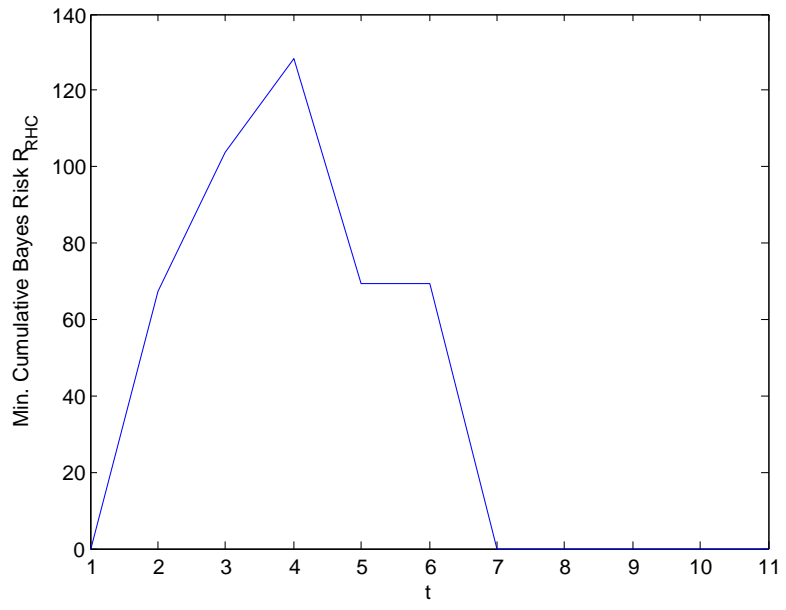

Figure 4: Minimum Bayes risk vs. time-step $t$. 
in (77). We assume the targets have a positional process noise parameter $\lambda_{t p}=1 e-3$, velocity positional process noise parameter $\lambda_{t v}=1 e-3$, and sensor positional process noise parameter $\lambda_{s p}=1.4 e-3$. The initialization of the targets and sensors is the same as those presented in subsection 6.1. The Figure 5 shows a simulation consisting of $M=4$ agents $N=4$ targets, $T=4$ RHC-step, $T^{\prime}=5$ particle step time horizon. The polygonal approximations used $n_{d}=6$ sides.

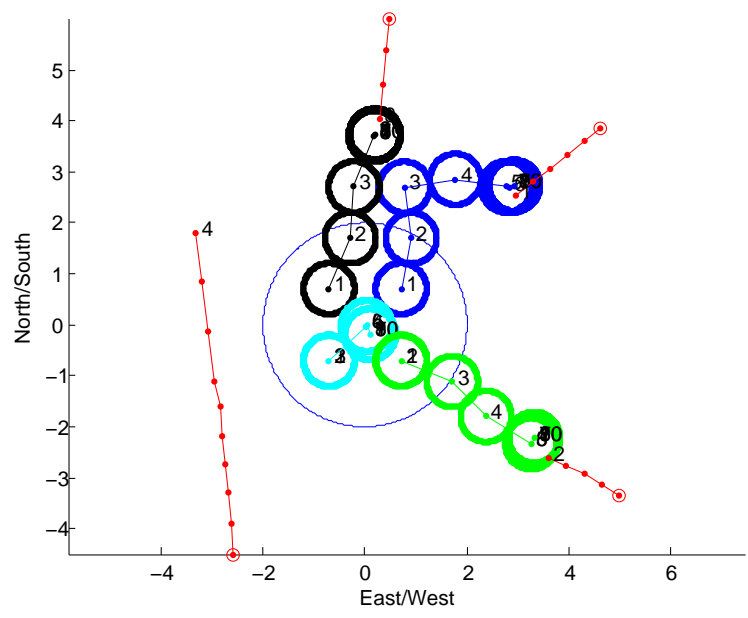

Figure 5: Particle Filter Simulation with $M=4, N=4, T=4, T^{\prime}=5, L=45$. Red lines are target tracks. Small circles are agent interception radii with agent paths in center. Large circle is region of protection.

The average time required to run an iteration of the simulation was 21.4 seconds, with an average MILP solve time of 0.57 seconds. Maximum time to perform an iteration of the simulation was 51.8 seconds, with maximum MILP solve time of 1.5 seconds. Example particle trajectories at time step $t=3$ are shown in the Figure 6. 


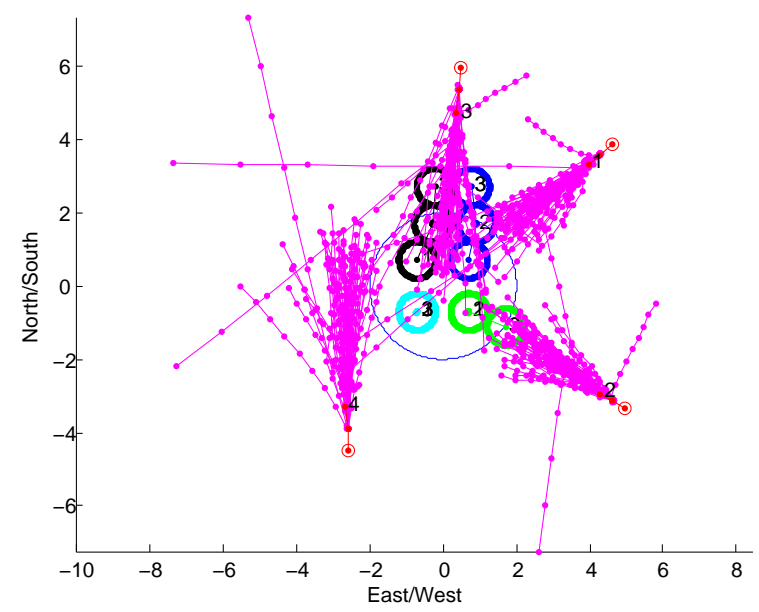

Figure 6: Particle filter simulation with $M=4, N=4, T=4, T^{\prime}=5, L=45, t=3$. Red lines are target tracks. Small circles are agent interception radii with agent paths in center. Large circle is region of protection. Purple lines are target particle projections.

\subsection{Collision Avoidance Simulation Results}

We now show a comparison of our framework with and without the collision avoidance risk cost function. We used the Cauchy random noise model discussed in 5.2. There were $M=4$ agents, $N=4$ targets, an optimization horizon of $T=4$, a threat horizon of $T^{\prime}=5$, and $L=25$ particles used in the simulation. A collision radius of $R_{c}=2.4$ was used. The probability of 
collision prior $P_{c}=0.2$. The costs for the collision risk were

$$
\begin{aligned}
& C_{0,0}^{\prime}=0 \\
& C_{1,0}^{\prime}=15 \\
& C_{0,1}^{\prime}=30 \\
& C_{1,1}^{\prime}=30 .
\end{aligned}
$$

Figure 7 shows a simulation without the collision avoidance risk in the receding-horizon control's cost function. As seen in Figure 7, the agents move within their interception radius of $R=1$ while intercepting the targets in the Northeast region, well in violation of the collision radius $R=2.4$. Figure 8 shows a simulation with identical starting positions for the targets and sensors, and includes the collision risk in the cost function. As seen in Figure 7, the sensors move in such a way that their interception radii do not intersect, as required by the collision radius $R=2.4$.

\subsection{Analysis of Costs Coefficients on Interception}

We have performed Monte Carlo simulations of our motion planning algorithm in order to determine the effect of varying the cost coefficients on target interception. The simulations involved 4 targets and 4 sensors, using the Kalman filter estimator from subsection 5.1. The targets start from the southern region of the area, at coordinates $[-2.5,-4.5],[-2.2,-4.5]$, $[2.5,-4.5]$, and $[2.5,-4.5]$. Depending on the stochastic noise, the targets may or may not enter the area. A typical simulation showing particle-based possible trajectories is shown in Figure 9. 


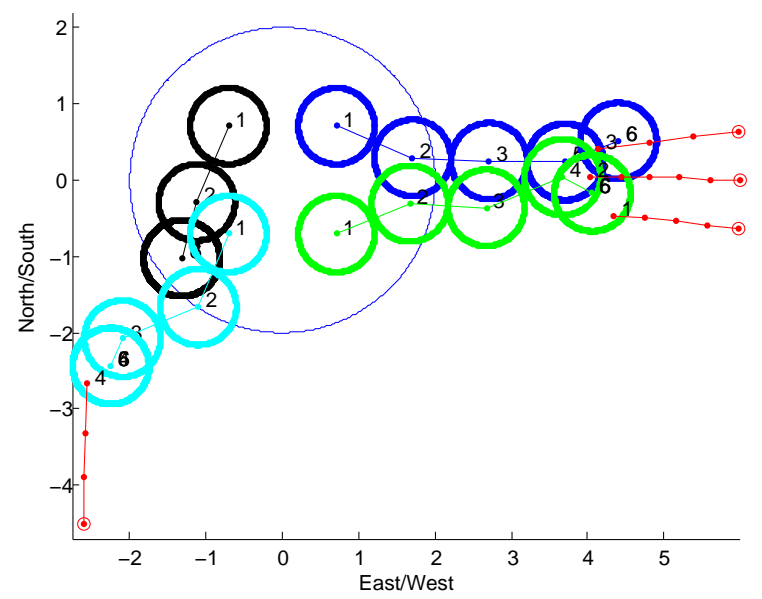

Figure 7: Simulation with no collision avoidance risk in cost function

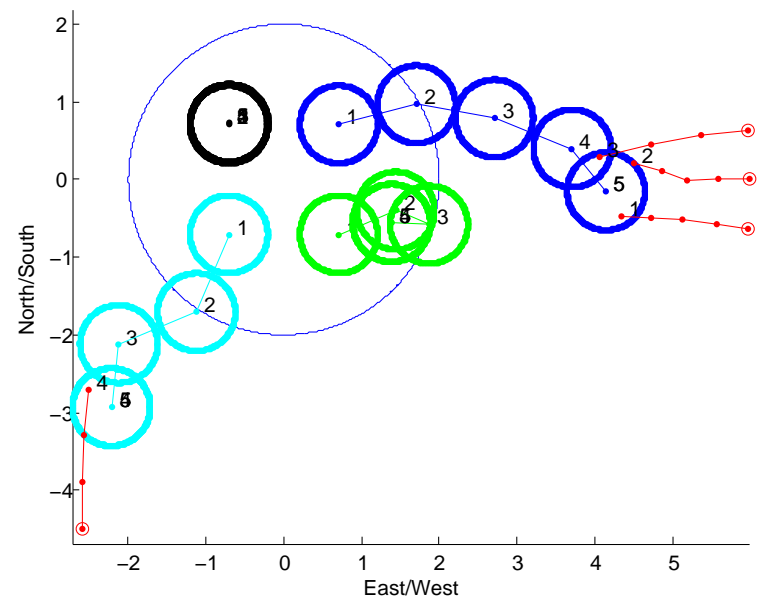

Figure 8: Simulation with collision avoidance risk in cost function 


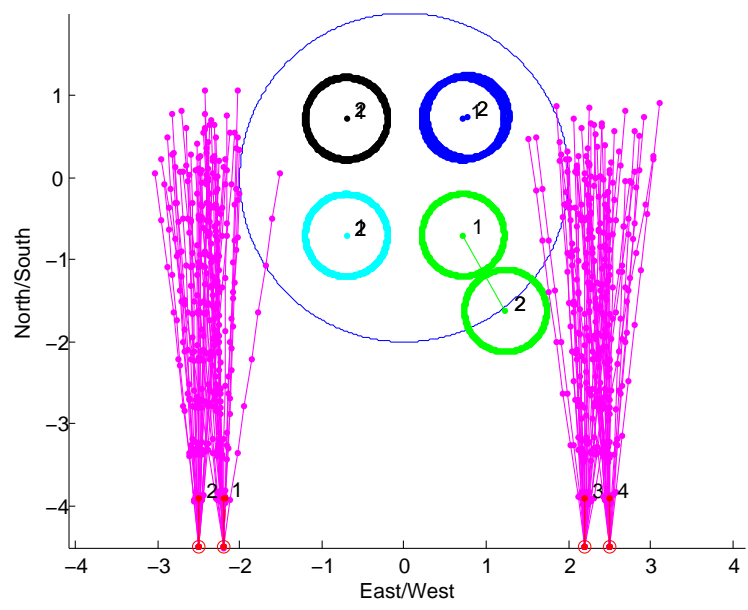

Figure 9: Monte Carlo simulation scenario

Table 1 shows the specific cost coefficients used and the average number of targets intercepted. For each cost coefficient pair, 30 simulations were run. As expected, the sensors intercept a decreasing number of targets as the ratio of missed-detection cost $C_{0,1}$ to false-alarm $\operatorname{cost} C_{1,0}$ decreases.

Table 1: Cost coefficients vs. average number of intercepted targets.

\begin{tabular}{|c|c|c|}
\hline $\begin{array}{c}\text { Missed-detection } \\
\text { cost } C_{0,1}\end{array}$ & $\begin{array}{c}\text { False-alarm } \\
\text { cost } C_{1,0}\end{array}$ & $\begin{array}{c}\text { Intercepted } \\
\text { target average }\end{array}$ \\
\hline \hline 100 & 1 & 2.90 \\
\hline 10 & 1 & 2.43 \\
\hline 5 & 5 & 1.13 \\
\hline 1 & 10 & 0.73 \\
\hline 1 & 100 & 0.63 \\
\hline
\end{tabular}




\section{CONCLUSION}

In this paper we have presented a novel extension of particle-based mixed integer linear programming techniques in order to reduce average or Bayes risk of multiple autonomous agents. This system incorporates uncertainty in both the state of the controllable agents, as well as uncertainty in the states and dynamics of the uncontrollable environment. We demonstrate the viability of this method to solve the target interception problem by posing the problem in a receding-horizon framework, as well as extending the framework to perform collision avoidance. By controlling the individual particles of the agent trajectories, the system optimizes the solution in order to minimize Bayes risk in a receding-horizon sense.

\section{ACKNOWLEDGMENT}

We gratefully acknowledge the helpful inputs for this work from Christian Reinl of Technische Universität Darmstadt, as well as the from the anonymous reviewers of the manuscript. This work was funded by the Office of Naval Research via grant N00014-08-1-0115, and the Dept. of Defense Sci-

ence, Mathematics, and Research for Transformation (SMART) fellowship program.

\section{References}

[1] H. V. Poor, An Introduction to Signal Detection and Estimation, Springer, 1994.

[2] S. Martnez, F. Bullo, Optimal sensor placement and motion coordination for target tracking, Automatica 42 (2006) 661 - 668. 
[3] R. R. Brooks, P. Eswaranramanathan, A. M. Sayeed, Distributed target classification and tracking in sensor networks, Proceedings of the IEEE 91 (2003) $1163-1171$.

[4] R. M. Murray, Recent research in cooperative control of multivehicle systems, Journal of Dynamic Systems, Measurement, and Control 129 (2007) 571-513.

[5] I. Hoballah, P. Varshney, Distributed Bayesian signal detection, IEEE Transactions on Information Theory 35 (1989) 995-1000.

[6] R. Viswanathan, P. K. Varshney, Distributed detection with multiple sensors i. fundamentals, Proceedings of the IEEE 85 (1997) 54-63.

[7] V. Goel, W. J. Byrne, Minimum bayes-risk automatic speech recognition, Computer Speech \& Language 14 (2000) 115-135.

[8] K. O. Perlmutter, S. Perlmutter, R. Gray, R. Olshen, K. Oehler, Bayes risk weighted vector quantization with posterior estimation for image compression and classification, IEEE Transactions on Image Processing 5 (1996) 347-360.

[9] L. Blackmore, S. Rajamanoharan, B. C. Williams, Active estimation for jump Markov linear systems, IEEE Transactions on Rob 53 (2008) $2223-2236$.

[10] S. L. Smith, S. D. Bopardikar, F. Bullo, A dynamic boundary guarding problem with translating targets, in: Joint 48th IEEE Conference on Decision and Control and 28th Chinese Control Conference. 
[11] N. Agmon, S. Kraus, G. A. Kaminka, Multi-robot perimeter patrol in adversarial settings, in: International Conference on Robotics and Automation.

[12] R. W. Beard, T. W. McLain, M. A. Goodrich, E. P. Anderson, Coordinated target assignment and intercept for unmanned air vehicles, IEEE Transactions on Robotics and Automation 18 (2002) 911-022.

[13] M. G. Earl, R. D'Andrea, Modeling and control of a multi-agent system using mixed integer linear programming, in: IEEE Conference on Decision and Control.

[14] G. Chasparis, J. Shamma, Linear-programming-based multi-vehicle path planning with adversaries, in: American Control Conference.

[15] A. Shende, M. J. Bays, D. J. Stilwell, Mixed integer linear programming for stochastic target interception, in: IEEE Conference on Intelligent Robots and Systems.

[16] J. Jansson, F. Gustafsson, A framework and automotive application of collision avoidance decision making, Automatica 44 (2008) 23472351.

[17] R. O. Duda, P. E. Hart, D. G. Stork, Pattern Classification, Wiley, 2001.

[18] C. Geddes, Algorithms for Computer Algebra, Kluwer Academic Publishers, 1992.

[19] A. Bemporad, M. Morari, Control of systems integrating logic, dynamics, and constraints, Automatica 35 (1999) $407-427$. 
[20] M. Bays, A. Shende, D. Stilwell, An approach to multi-agent area protection using Bayes risk, IEEE International Conference on Robotics and Automation (2012).

[21] L. Blackmore, M. Ono, A. Bektassov, B. C. Williams, A probabilistic particle-control approximation of chance-constrained stochastic predictive control, IEEE Transactions on Robotics 26 (2010) 502-517.

[22] L. C. Yang, J. K. Kuchar, Prototype conflict alerting system for free flight, Journal of guidance, control, and dynamics 20 (1997) 768-776.

[23] J. R. Babbitt, Order 8020.11C: Aircraft Accident and Incident Notification, Investigation, and Reporting, Federal Aviation Administration, 2010 .

[24] Busygin et al, Handbook on Modeling for Discrete Optimization, Springer, 2006.

[25] S. Y. Shatskikh, Multivariate cauchy distributions as locally gaussian distributions, Journal of Mathematical Sciences 78 (1996) 102-108.

[26] M. Ikoma, N. Ichimura, T. Higuchi, H. Maeda, Maneuvering target tracking by using particle filter, in: International Fuzzy Systems Association World Congress.

[27] D. Crisan, A. Doucet, A survey of convergence results on particle filtering methods for practitioners, IEEE Transactions on Signal Processing 50 (2002) 736-745. 
[28] B. Ristic, S. Arulampalam, N. Gordon, Beyond the Kalman Filter, Artec House, 2004.

[29] G. Kitagawa, Monte Carlo filter and smoother for non-gaussian nonlinear state space models, in: Journal of Computational and Graphical Statistics.

[30] C. A. Floudas, Nonlinear and Mixed-Integer Optimization: Fundamentals and Applications, Oxford University Press, 1995.

[31] A. Richards, J. How, Mixed-integer programming for control, in: American Control Conference.

[32] A. Richards, Y. Kuwata, J. How, Experimental demonstrations of realtime milp control, in: AIAA Guidance, Navigation, and Control Conference.

[33] K. Culligan, M. Valenti, Y. Kuwata, J. P. How, Three-dimensional flight experiments using on-line mixed-integer linear programming trajectory optimization, in: American Control Conference.

[34] D. Mellinger, A. Kushleyev, V. Kumar, Mixed-integer quadratic program trajectory generation for heterogeneous quadrotor teams, in: International Conference on Robotics and Automation.

[35] S. Tang, V. Kumar, Mixed integer quadratic program trajectory generation for a quadrotor with a cable-suspended payload, in: IEEE International Conference on Robotics and Automation. 
Dr. Matthew Bays is a research engineer at the Naval Surface Warfare Center, Panama City Division. He obtained a B.S. in Mechanical Engineering from Cornell University, and M.Eng. and Ph.D. degrees in Mechanical Engineering from Virginia Tech. His research interests include optimization, multi-agent motion control, and statistical decision theory.

Dr. Apoorva Shende is Assistant Vice President at Nomura. Dr. Shende obtained a B.S. in Civil Engineering from IIT Bombay, and M.S. and Ph.D. degrees in Engineering Science \& Mechanics from Virginia Tech. His research interests include numerical analysis, modeling, and optimization.

Dr. Daniel J. Stilwell is a Professor at the Bradley Department of Electrical \& Computer Engineering at Virginia Tech. He obtained a B.S. in Electrical Engineering from the University of Massachusetts at Amherst, and M.S. and Ph.D. Degrees in Electrical Engineering from Johns Hopkins University. His research interests include robotics, control theory, and estimation. 
Matthew J. Bays

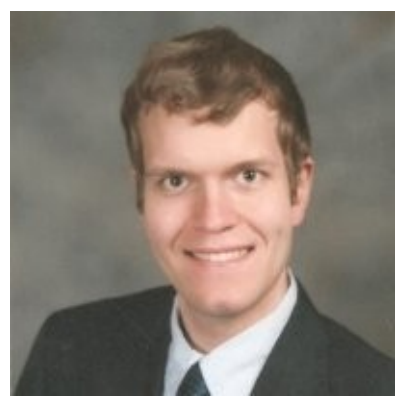

Apoorva Shende

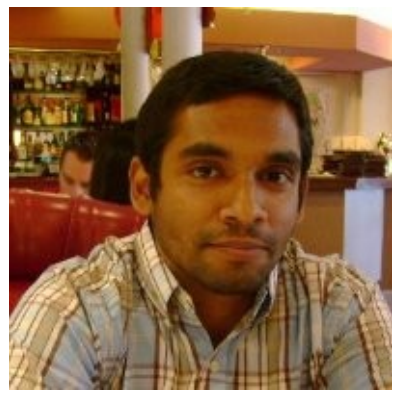

Daniel J. Stilwell

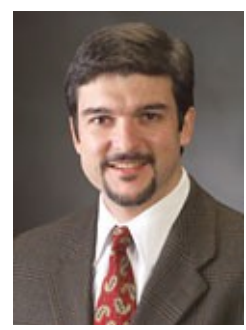

